\section{A general analysis of variance package}

\section{PAUL W. FLAGG \\ Dartmouth College, Hanover, New Hampshire 03755}

Analysis of variance is one of the most commonly used statistical tests in the social sciences, especially psychology. This test is appropriate for many different experiment designs; however, except for simple designs, the computations required are often staggering. Of course, with the advent of the computer, most schools have access to an analysis of variance program of some type. However, many of these programs are either exceedingly complex to use and/or do not perform all the calculations that might be desired. For example, the analysis of variance routines offered by IBM calculate only the sum of squares for a complete factorial design, while those described by Jernstedt (1972) can only handle designs with a maximum of three between and one within factor. The main analysis of variance program in this package, on the other hand, has a fairly straightforward input format, calculates $F$ ratios and their cxact probabilities for any design of up to 14 factors (any number of which may be random) that has equal cell sizes. Such useful information as the proportion of variance accounted for by each effect and quasi-Fis are also calculated. As well, the other two programs in the package aid the user in performing a priori and post-hoc tests on the means of an analysis of variance.

Description. The main program, called ANOVAR4, performs an analysis of variance on a complete factorial design with equal cell sizes, or any design that can be considered as such. The program can handle designs with up to 14 factors where subjects or replications is considered a factor; on the Dartmouth time-sharing system (DTSS), the practical limit is eight factors. Possible designs include: complete factorial (fully crossed), complete factorial with replications, completely nested, and partially nested designs. The nature of the design is specified by the use of a standard notational format. Any number of factors may be specified as random, thus allowing completely fixed, completely random, and mixed designs. At run time the user has the option of obtaining: the complete factorial sum of squares table, the revised sum of squares table (where terms have been pooled as determined by the design), and the expected mean squares table. The results table is always printed. When there is only one random factor, the results table will contain the mean squares and degrees of freedom for each effect, the $F$ ratio, the exact probability of $F$, and the proportion of variance accounted for by each effect. When there is more than one random factor, the proportion of variance accounted for by each effect is not given; however, where appropriate, quasi-Fs will be calculated.
The second program, MEANS4, will take as input the same data as ANOVAR4 and return means and standard deviations for user-specified main effects and interactions. The final program, COMPARE, makes use of information supplied by the other two programs to perform planned comparisons, trend, residual, Scheffé, and Tukey tests.

Input. The programs ANOVAR4 and MEANS4 work with data located either in an input file or in data statements within the program. The data may be read into the input file from punched cards or tape (magnetic tape) or entered from any Teletype-format input device. The data to be analyzed must contain a few header lines specifying how many factors are in the design, the design itself, what factors are random, and levels per factor. The data then follow in the order specified by the design. COMPARE is entirely run-time interactive and requests needed information from the user (e.g., means, weights, error term, etc.).

Computer and Language. These programs are written in BASIC and have been used on a Honeywell 635 system in both interactive time-sharing mode and batch processing mode. The use of these programs on other systems depends on the size of the design, especially with respect to ANOVAR4 (the larger the design, the more core memory required). In calculating the sums of squares and degrees of freedom, ANOVAR4 makes use of the IBM SSP routines AVDAT, AVCAL, and MEANQ. These were translated from FORTRAN to BASIC by G. C. Jemstedt. The other computational algorithms used by ANOVAR4 and COMPARE are modeled after those given by Lindman (1974) and Winer (1971). These programs contain extensive internal documentation describing use and operating principles.

Availability. A listing of the programs is available from the author upon request.

\section{REFERENCE NOTE}

1. IBM. System 360 scientific subroutine package (360A(M.O3X) progranmers manual. Manual No. GH20-0205.

\section{REFERENCES}

Jernstedt. G. C. Broad-range analysis of variance. Behavioral Science. 1972, 19, 397-398.

Lindman, H. R. Anulysis of variunce in complex experimental designs. San Francisco: W. H. Freeman. 1974.

Winf. R. B. J. Statistical principles in experimental design. 2nd ed. New Yurk: MeGraw-Hill. 1971 